\title{
Soziale Arbeit als weißer* Raum - eine Critical Whiteness Perspektive auf die Soziale Arbeit in der postmigrantischen Gesellschaft
}

\author{
Martina Tißberger
}

Online publiziert: 27. April 2020

(C) Der/die Autor(en) 2020

Zusammenfassung Viele Einrichtungen der Sozialen Arbeit stellen nach wie vor weiße* Räume in einer (Post-)Migrationsgesellschaft dar. Dieser Text geht der Frage nach, warum das so ist. Er gibt zunächst einen historischen Überblick zum Umgang mit migrationsbedingter Differenz, der zeigt, dass die Themen Rassismus und Machtverhältnisse lange Zeit eine Leerstelle bildeten, die von den Critical Whiteness Studies nun adressiert wird. Diese richten den Blick auf die weiße* Dominanzkultur und legen offen, wie ,das ganz unverdächtige, wohlmeinende Subjekt in der Mitte der Gesellschaft" unbewusst Rassismus re/produziert. Anschließend wird eine qualitative Untersuchung zur Bedeutung von Whiteness in der Sozialen Arbeit dargestellt, die über narrative Interviews mit Sozialarbeiter*innen analysiert, wie diese dazu beitragen, Soziale Arbeit zu einem weißen* Raum zu machen und sich selbst zu Kompliz*innen der Kolonialität von Macht. Soziale Arbeit als Menschenrechtsprofession kann jedoch aus dieser mandatswidrigen Situation herausfinden.

Schlüsselwörter Critical Whiteness · (Post-)Migrationsgesellschaft ·

Intersektionalität · Koloniale Episteme 


\title{
Social work as a white* space-a critical whiteness perspective on social work in a postmigrant society
}

\begin{abstract}
Many social work institutions still represent white* spaces in (post-)migration societies. This text looks at why. First of all, a historical overview of the handling with migration-related differences is given, which shows that the issues of racism and power relations have long been a void. This is followed by an introduction to critical whiteness studies, which address this void, look at the white* culture of dominance and reveal how "the completely unsuspicious, well-meaning subject in the middle of society" unconsciously re/produces racism. A qualitative study of the importance of whiteness in social work is then presented. Using narrative interviews with social workers as an example, we analyze how they help to make social work a white* space and become accomplices of the coloniality of power. Finally, it shows ways in which social work, as a human rights profession, can find out from this non-mandated situation.
\end{abstract}

Keywords Critical Whiteness · (Post)Migration Society · Intersectionality · Colonial Episteme

\section{Zur Geschichte des Umgangs mit postmigrationsgesellschaftlichen Differenzverhältnissen}

Die Soziale Arbeit in deutschsprachigen Gesellschaften kann nach wie vor als weißer*1 Raum bezeichnet werden. Auch wenn sich gerade für diese Disziplin als Menschenrechtsprofession viele Menschen mit Migrations- und Rassismuserfahrung interessieren und das Fach studieren, praktizieren oder in Forschung und Lehre tätig sein wollen, wird das Feld von Weißen* dominiert. Dieser Umstand wird jedoch de-thematisiert. Erst langsam entwickelt sich ein kritischer Diskurs über den strukturellen Rassismus in der Sozialen Arbeit. Dass dieser kritische Diskurs im internationalen und europaweiten Vergleich relativ spät stattfindet, hängt unter anderem mit dem Nationalsozialismus (NS) zusammen. Nach 1945 schien es, als säße der Schock über den faschistischen Rassenwahn gesellschaftlich so tief, dass man über Rassismus nicht mehr sprechen muss. Es fand daher in Deutschland bis in die 1960er Jahre keine nennenswerte Aufarbeitung statt und auch danach wurde Rassismus nur als Problem am Rande der Gesellschaft gesehen, mit „Täter*innen“ aus Kreisen des Rechtsradikalismus und „Opfern“, die als „Ausländer*innen“ betrachtet

\footnotetext{
1 Sämtliche Begriffe, die sich auf die sozialen Konstruktionen Geschlecht* und Rasse* beziehen, sind in diesem Text mit einem Asterisk gekennzeichnet. Diese Praxis des Gender-Gap - eine linguistische Strategie zur Überwindung von Heteronormativität und Sexismus - wird auf Rasse* übertragen, um beider Konstruktionscharakter gerecht zu werden und Ontologisierungseffekte durch Sprache zu vermeiden. Das Sternchen am Ende von Begriffen wie Frau*, schwarz*, weiß* oder männlich* soll deutlich machen, dass diese Begriffe zur vergeschlechtlichenden und rassifizierenden Markierung dienen und nicht eine Qualität, „Eigenschaft“ oder das „Wesen“ von Subjekten bezeichnen. Die ontologisierende Wirkung von Rasse* und Geschlecht* ist das Problem und Gegenstand der Gender- und Critical Whiteness Studies: die Frage, wie die einzelnen Subjekte die Ontologisierung von Rasse* und Geschlecht* (unbewusst) reproduzieren.
} 
wurden. In Österreich fühlt man sich bis heute nicht verantwortlich für den Rassismus des NS und positioniert sich politisch als dessen Opfer, obgleich die Spuren des Austrofaschismus in der Politik bis in den zeitgenössischen Rechtspopulismus hinein reichen. Das Neutralitätsprinzip der Schweiz wurde während des Zweiten Weltkriegs durch Kooperationen mit dem NS unterlaufen und in der deutschsprachigen Schweiz gab es nicht wenige Anhänger*innen von Hitlers Politik. Aber auch dort spielte die Aufarbeitung nach 1945 keine nennenswerte Rolle.

Bis zum 21. Jahrhundert waren die Begriffe Rasse* und Rassismus im gesellschaftlichen Diskurs tabu. Der Versuch, mit dieser Tabuisierung die Bedeutung der Kategorie Rasse* für das allgemeine Denken zu beenden, scheiterte. Rassismus blieb omnipräsent, nur gab es keine Sprache mehr, um ihn adäquat zu adressieren. Die bevorzugt verwendeten Begriffe „Fremdenfeindlichkeit“ oder „Ausländer*innenfeindlichkeit" beschreiben nicht, was passiert. Zielscheibe von gewaltvollen Angriffen oder Alltagsrassismus sind nicht weiße* Schwedinnen oder weiße* Kanadier, sondern Afrodeutsche, ,albanische Österreicher*innen“ oder „türkische Schweizer*innen". Grund der Ausgrenzung und Feindseligkeit ist nicht die vermeintliche Fremdheit, sondern die Imagination einer gefährlichen Differenz, welche durch phänotypische oder kulturalistisch-rassifizierende Merkmale wie Akzent, ein nicht Deutsch klingender Name oder eine nicht christlich(-säkularisierte) Religionszugehörigkeit markiert wird - kurzum: Rasse*.

Gemäß den Rassen*theorien des 18. und 19. Jahrhunderts wird nach wie vor imaginiert, dass Weißsein* bedeutet, (west- und nord-)europäisch zu sein. Menschen, die zwar eine weiße* Hautfarbe haben, aber beispielsweise „slawisch“, muslimisch oder jüdisch sind, werden dieser Ordnung zufolge als ,off-white“ imaginiert. Schwarze* stellen in dieser rassistischen Skala den Ausgangspunkt als „Primitivität“ und Gegenpol zu Weißen* dar; Letztere gehen aus dieser Ordnung als Inbegriff der Zivilisation hervor.

Die pädagogischen Konzepte für den Umgang mit (post-)migrationsgesellschaftlichen Differenzverhältnissen spiegeln die De-Thematisierung des Rassismus wider. Das erste dieser Konzepte war die sogenannte Ausländer*innenpädagogik, die in den 1970er Jahren in Reaktion auf die Erkenntnis entwickelt wurde, dass die Kinder der sogenannten Gastarbeiter*innen, die ab der Mitte der 1950er Jahre in Deutschland und ab Anfang der 1960er Jahre in Österreich angeworben wurden, in den Bildungseinrichtungen nicht länger ignoriert werden konnten. Der kompensatorische, defizitorientierte und assimilatorische Ansatz dieser Pädagogik (vgl. Mecheril 2010; Nohl 2006, S. 17 ff.; Krüger-Potratz 2005, S. 121 ff.) geriet in den 1980er Jahren in die Kritik und wurde in den 1990er Jahren durch die sogenannte „Interkulturelle Pädagogik" abgelöst.

Es stellte sich jedoch schnell heraus, dass sich das Differenz-als-Problem-Denken in die „Interkulturalität“ hinein verlängert hatte. Statt, wie es der Begriff suggeriert, das „Zwischen“ den „Kulturen“ in Form von Austausch und Entwicklung sowie die Potenziale und Chancen, die durch Differenzen, aber auch Gemeinsamkeiten entstehen (vgl. Terkessidis 2010), zu adressieren, wurden - und werden immer noch - „Kulturen“ homogenisiert, als statische Entitäten begriffen und hierarchisch angeordnet. Wie selbstverständlich und oft gänzlich unreflektiert wird dabei von der „abendländischen“, christlich-säkularisierten „Kultur“ als Norm ausgegangen, von 
der alle anderen „Kulturen“ abweichen und als solche „besondert“ und ihre Subjekte „,verandert“ werden. Das Erfolgsprogramm „Interkulturalität“ gipfelt konzeptionell in der „Interkulturellen Kompetenz“, die Teilnehmer*innen - meist der normativ dominanten „Kultur“ angehörend - in entsprechenden Lehrgängen von Volkshochschulkursen bis zu universitären Studiengängen durch vermeintliches Wissen über die „Anderen“ Gewissheiten über den Umgang mit migrationsbedingter Differenz suggeriert. „Interkulturalität“ ist zur Strategie geworden, euro- und androzentrische Dominanzkulturen (Rommelspacher 1995) zu erhalten, ohne sich dem Vorwurf auszusetzen, eben dies zu tun - ist doch „Interkulturalität“ zur „Querschnittskompetenz“ in der Bildungslandschaft dieser „Kulturen“ geworden (kritisch dazu u. a. Hamburger 2012; Castro Varela 2012; Freise 2005; Eppenstein 2003). Kratzt man an der Oberfläche von Diskursen über Kultur oder Ethnizität, taucht meist Rasse* darunter auf (Seshadri-Crooks 2000); „Kultur“ ist zum „Sprachversteck“ (Leiprecht 2001) für Rassismus geworden.

Bis ins 21. Jahrhundert hinein war der Mainstream interkultureller Konzepte durch die Ausblendung von Rassismus und Machtverhältnissen gekennzeichnet und trug dadurch zu deren Stabilisierung bei. Inzwischen gibt es eine Reihe neuer Ansätze, die die Defizite der Interkulturalitätskonzepte überwinden wollen und auch den Begriff hinter sich lassen, etwa Social Justice (Czollek et al. 2009), Rassismuskritik (Melter und Mecheril 2009), Diversitätsbewusste Pädagogik (Leiprecht 2011) oder Postmigrantische Perspektiven (Foroutan et al. 2018; Yildiz und Hill 2015; Foroutan 2013; Langhoff 2011). Die Critical Whiteness Studies gehören zu diesen Ansätzen, wobei sie sich weniger als Konzept denn als kritische Perspektive verstehen. Der Begriff der postmigrantischen Gesellschaft verweist u. a. auf die Tatsache, dass viele Menschen als Migrant*innen bezeichnet werden, die gar keine Migrationserfahrung haben, sondern in Deutschland, Österreich oder der Schweiz geboren und aufgewachsen sind, deren Vorfahren jedoch migriert sind. Der Begriff verweist also auf das Problem der mangelnden ,Integrationsbereitschaft“ der weißen* Dominanzkultur aufgrund ihres Rassismus. Da in diesem Text die Situation von Migrant*innen, aber auch „Post-Migrant*innen“ Thema ist, wird auch die Schreibweise ,(post-)migrantisch" verwendet.

\section{Critical Whiteness}

Critical Whiteness ist im Nexus der Auseinandersetzungen mit Rassismen und Feminismen als Theorie und Praxis hegemonialer Selbstreflexion entstanden und stellt im Kern ihres akademischen Feldes - der Critical Whiteness Studies - eine Epistemologiekritik dar. Die Critical Whiteness Studies markieren einen Paradigmenwechsel in der Rassismusforschung. Von denjenigen angestoßen, die als Objekte und „Opfer“ des Rassismus galten, richtet sich der Finger, der bisher auf sie und Täter*innen in Form von Rechtsradikalen zeigte, nun auf die Subjekte des Rassismus - auf diejenigen, die ihn perpetuieren und zwar oft wider Willen und trotz besseren Wissens. Im Fokus steht also das ganz unverdächtige weiße* Subjekt in der Mitte der Gesellschaft. Der vorliegende Text adressiert dementsprechend genau dieses Subjekt und nimmt seine Perspektive ein. Es ist die Aufgabe von Weißen*, Rassismus zu über- 
winden, denn sie sind seine Protagonist*innen und die ultimativen Profiteur*innen des Rassismus als Gesellschaftsstruktur. Die Critical Whiteness Studies untersuchen die konstitutive Bedeutung des Rassismus für die Genese und Aufrechterhaltung der Struktur der (westlichen) Gesellschaften. So sind auch die Institutionen der Sozialen Arbeit bedeutende Räume, in denen Rassismus produziert und perpetuiert wird, oder aber adressiert, durchkreuzt und überwunden werden kann.

Den Unterschied zwischen den Critical Whiteness Studies und antirassistischen Ansätzen mag ein einfaches Beispiel veranschaulichen. Es ist für Weiße* einfach zu sagen: „Ich bin nicht rassistisch“ und das mit Argumenten zu unterlegen, wie etwa die Aussage: „Ich habe „türkische“ und schwarze* Freund*innen, engagiere mich bei Amnesty International und setzte mich kürzlich für die Einstellung einer „asiatisch-deutschen“ Bewerberin ein.“ Das Problem sind demnach also die rassistischen institutionellen oder sozialen Strukturen - andere Personen -, aber nicht ich. Aus dieser Position heraus ist es für Weiße* möglich, nichts mit Rassismus zu tun zu haben, also „unschuldig“ zu sein. Im Unterschied dazu wäre es absurd für Weiße* zu sagen: „Ich bin nicht weiß*.“ Letzteres bedeutet, dass auch wenn ich mich für eine rassistisch markierte ${ }^{2}$ Kollegin engagiere, mich niemand aufgrund von Rassekonstruktionen bei einem Bewerbungsverfahren diskriminieren wird. Für mich bleiben keine Türen aufgrund einer rassistischen Markierung verschlossen; niemand belästigt mich mit Fragen nach meiner Herkunft oder „,verandert“ mich in sonst einer Weise. Weiße* profitieren permanent vom strukturellen Rassismus der Gesellschaft und sind damit - wenn auch wider Willen - Teil des Rassismus, also „schuldig“.

Die Auseinandersetzung mit dem eigenen Weißsein* und mit Whiteness als machtvollem Signifikanten des kulturellen Symbolischen innerhalb der rassistischen Dominanzkulturen geht also über einzelne antirassistische Aktivitäten hinaus. Es gibt keine „Komfortzone“ mehr. Genauso wie alle rassistisch markierten Menschen, die jederzeit wahrnehmen, wo sie offensichtlich oder subtil ausgeschlossen und ,verandert" werden, nehmen auch diejenigen, die im Rassismus bisher de-markiert waren - die als neutral galten - wahr, in welcher Weise sie durch ihr Weißsein* ermächtigt und Nicht-Weiße* diskriminiert werden. George Yancy (2004) schreibt, dass Whiteness so lange ,schuldig“ ist, wie es ein Ensemble von Machtverhältnissen konstitutiert, in denen Weiße* mit Macht und Vorteilen gegenüber Nicht-Weißen* ausgestattet sind (vgl. Yancy 2004, S. 6). Eben diese Machtverhältnisse werden von denjenigen, die von ihnen profitieren, gerne ausgeblendet. Erst wenn diejenigen, die im Rassismus de-markiert sind, ihr Weißsein* als Markierung wahrnehmen, können sie ein Bewusstsein für dessen Bedeutung und damit die Voraussetzung für Handlungsfähigkeit entwickeln - vorausgesetzt natürlich, sie wollen das überhaupt.

\footnotetext{
2 Rassismus ist ein Akt der (De-)Markierung. Ich bezeichne die Markierung als rassistisch und nicht etwa „rassisch“, weil es der mentale Akt der Markierung ist, der Rasse* entstehen lässt. Der mentale Akt des Markierens ist rassistisch. So ist in diesem Text nicht die Rede von Migrant*innen oder gar „Ausländer*innen“, sondern wird die Bezeichnung ,rassistisch (oder migrantisch) markiert“ für alle Menschen verwendet, die von der Dominanzkultur rassistisch behandelt werden.
} 


\section{Whiteness - Artikulationen in den Codes der aufklärerischen Moderne}

Whiteness wird als strukturierendes Moment einer Herrschaftsdimension - einer rassistischen Matrix - verstanden, von der aus die Subjekte einer Gesellschaft an der Norm des Weißseins* gemessen, markiert und positioniert werden, wobei das Weißsein* als Norm unbenannt, unsichtbar und de-thematisiert bleibt. Whiteness ist so Signifikant für alles, was die abendländische Moderne zum Ideal erklärt hat; dem gegenüber sind die ,dunkleren Anderen“ mit allen gegenteiligen Merkmalen ausgestattet. In beliebigen weißen* dominanzkulturellen Verhältnissen kann beobachtet werden, dass Nicht-Weiße* mit negativen Eigenschaften bedacht werden und der Diskurs über Einwanderung sie entsprechend ,,anschwärzt"; der Wohlstand der Weißen* und der Status einer Gesellschaft scheinen immer bedroht durch die Einwandernden. Die geringsten Unterschiede zwischen „Autochthonen“ und „Allochthonen“ können zur Rassifizierung - der Ontologisierung kultureller Differenzen - führen. Whiteness liegt im Symbolischen und ist daher in unterschiedlicher Weise materialisierbar. Es bedarf nicht der ,weißen Hautfarbe“, sondern der Bezugnahme auf das Ideal der aufklärerischen Moderne mit ihrer imperialistischen Episteme - es bedarf des symbolischen Kapitals dieser Episteme, um andere Andere re/produzieren zu können. Whiteness als Signifikant des kulturellen Symbolischen des Rassismus artikuliert sich daher selten in offen rassistischen Formen, sondern vielmehr in den Codes des Aufklärerischen, Paternalistischen, Emanzipativen und in Entwicklungsnarrativen. Wir können hier bereits erahnen, wie sehr die Soziale Arbeit gefährdet ist, Komplizin der Kolonialität der Macht (Quijano 2016) zu werden.

Rasse* ist eine soziale Konstruktion, das heißt, sie ist auf ihre permanente Re/ Produktion angewiesen. „Weißsein“ und „Schwarzsein“, um die beiden Extrempole der Konstruktion Rasse* zu bezeichnen, sind Effekte des Rassismus. Diese zu benennen, ist wichtig. Sie als Ausgangspunkt von Untersuchungen zum Rassismus und von Praktiken gegen Rassismus zu nehmen, hieße aber, das Wesentliche des Rassismus, nämlich die Mechanismen seiner Re/Produktion auszublenden: das Aufteilen der Menschheit in Gruppen und ihre hierarchische Anordnung - die Ontologisierung von Menschen entlang rassistischer Kategorien. Whiteness bezeichnet mehr als ontologisierte Subjektpositionen wie „Weißsein“ oder „Schwarzsein“. Whiteness ist eine historisch gewordene Struktur, die aus konkreten politischen, ökonomischen und sozialen Machtkonstellationen heraus entstanden ist und die sämtliche Bereiche des Lebens durchzieht. Als solche ist sie eine Machtstruktur, von der Subjektivierung ausgeht. Sie ist eine Macht, die ihren Namen nicht nennt. Sie ist zusammen mit dem Rassismus entstanden, als sein de-thematisiertes Machtzentrum - eine wirkmächtige Imagination, die kollektiv wie individuell wirkt, meist unbewusst. Als solches ist Whiteness nicht ins Deutsche zu übersetzen.

\section{Whiteness an der Schnittstelle von Rassismus und Gender}

Die Vorläufer der Critical Whiteness Studies können bis ins 19. Jahrhundert zurückverfolgt werden, etwa zu dem afroamerikanischen Philosophen und Soziologen 
W. E. B. DuBois und der afroamerikanischen Abolitionistin und Frauenrechtlerin Sojourner Truth, die beide öffentlich Rassismuskritik formulierten. S. Truth, die sich zugleich für die Abschaffung der Sklaverei (in der sie selbst noch gelebt hatte) und für das Frauenwahlrecht in den USA einsetzte, brachte in einer berühmt gewordenen Rede bereits 1851 mit ihrer Frage „Ain't I a woman?“ das Thema der Intersektionalität pointiert zum Ausdruck. Sie verdeutlichte ihren weißen* Mitstreiter*innen, dass sie in ihrem Kampf für die Frauen*rechte längst nicht alle Frauen* berücksichtigten und sie zeigte ihnen mit zahlreichen Beispielen aus ihrem Leben als schwarze* Frau* - ehemalige Sklavin -, dass es sehr unterschiedliche Existenzweisen von Frauen* gibt und dass Rassismus weiße* und schwarze* Frauen* strukturell trennt. Wie bereits die Aufklärer, die in ihrem universalistisch begründeten bürgerlichen Freiheitsbegriff lediglich weiße*, besitzende (heterosexuelle) Männer* bedachten, hatten auch die Protagonist*innen der amerikanischen Frauenrechtsbewegung das Weißsein* dieser Frauen* und ihrer Rechte zwar als Norm gesetzt, diesen Umstand dann aber gleichzeitig ausgeblendet. Die erste US-amerikanische Frauen*bewegung war im Zusammenhang mit dem Abolitionismus entstanden; sie gründete also in der Intersektion von Rassismus und Gender, ein konstitutiver Zusammenhang, der kaum epistemologische Berücksichtigung fand.

Toni Morrisons (1994) Literaturkritik „Im Dunkeln spielen“, gilt als ein Gründungstext der Critical Whiteness Studies. Sie analysiert in dieser Essay-Sammlung das literarische „Spielen im Dunkeln der Moderne“ anhand literarischer Werke. T. Morrison geht empirisch vor und untersucht die Leerstellen in den Texten - das, was nicht gesagt wird, was sie allerdings als schwarze* Leserin und Autorin als abwesende Anwesenheit wahrnimmt, jenes Schattendasein, das etwa Schwarze* in den USA als Sklav*innen und als Nachfahren von Sklav*innen zu führen verdammt waren und sind. Die Subjekte dieser Texte wie sämtliche ihrer Autor*innen sind weiß* und wir wissen es, weil es nicht gesagt wird (Morrison 1994, S. 104). Das Ding hingegen, das in all den Texten auftaucht, ist eine afrikanistische Präsenz oder Persona, eine beunruhigende Population, die als konstitutives Außen, als Nicht-Ich - als Negativ - die Formierung des Ichs dieser Texte ermöglicht.

Ein anderes wichtiges Werk der Critical Whiteness Studies ist Richard Dyers Buch „White“ (1997), in dem der Filmtheoretiker anhand der visuellen Kultur herausarbeitet, wie Whiteness (historisch) durch Repräsentation zum symbolischen Zentrum westlicher Kulturen wurde und in welcher Weise Sexualitäten in diese rassistische Figuration eingearbeitet sind. Er zeigt, wie in der visuellen Kultur NichtWeißsein*, Weiblichkeit* und „,deviante Sexualität“ zum konstitutiven Außen einer mit Geist, Göttlichkeit, heteronormativer Männlichkeit* und Erhabensein ausgestatteten Whiteness wird, die ihre Wirksamkeit auch darüber entfaltet, dass sie dethematisiertert bleibt. Das Werk wurde zum zentralen Referenzpunkt für die Critical Whiteness Studies, weil R. Dyer (1997) in seiner Analyse die epistemische, symbolische und materiale Dimension von Whiteness erfasst. Dabei macht er theoretische Anleihen bei der Psychoanalyse wie bei der Diskursanalyse und wendet deren methodische Instrumentarien an. Wenige nachfolgenden Arbeiten haben in solch umfassender und detaillierter Weise das Phänomen Whiteness beschrieben.

Ruth Frankenbergs (1993) Arbeit „White Women, Race Matters: The Social Construction of Whiteness“" war die erste empirische Studie, die sich explizit mit der 
Phänomenologie von Whiteness beschäftigte und daher das Feld der Critical Whiteness Studies mitbegründete. In dieser damals sehr innovativen Untersuchung wollte R. Frankenberg das Terrain von Whiteness ,explorieren“ und „kartographieren“. R. Frankenberg stellt fest, dass Rasse* für das Leben weißer* Frauen* in den USA nicht nur aufgrund von strukturellem Rassismus Bedeutung gewinnt. Die von ihr interviewten Frauen* nahmen zwar unwillkürlich ihre unbenannten und unmarkierten Orte in den ,rassistischen Landschaften“ ein, in die sie hinein geboren wurden, aber sie reproduzierten diese Orte auch aktiv. Es sind die Subjekte selbst, welche die Lokalisierung von Privilegien ${ }^{3}$ und die Perpetuierung der Normativität weißer* Kultur vornehmen - allerdings meist unbewusst. Ein ,undoing of whiteness“ sei deshalb nicht nur Angelegenheit der Politik, sondern der einzelnen Individuen, so das Resümee dieser Studie.

Was dem Bewusstsein zugänglich ist, kann verändert werden. Whiteness scheint allerdings genau dort am wirkmächtigsten, wo es sich dem Bewusstsein entzieht. Shannon Sullivan (2006) schreibt dazu: „White privilege functions best when it appears not to be functioning at all [...] The flashy obviousness of white supremacy will be its downfall in a ,civilized“ world that prides itself on its democratic tolerance and inclusiveness“ (Sullivan 2006, S. 187).

Im deutschsprachigen Raum gaben Katharina Oguntoye, May Opitz (später May Ayim) und Dagmar Schulz (1986) das Buch „Farbe bekennen“ heraus, in dem afrodeutsche Autorinnen ihr Leben und den Rassismus, von dem es geprägt war, beschrieben. Die Autorinnen thematisierten dort bereits den deutschen Kolonialismus, der bis ins Jahr 2004 - der einhundertsten Jährung des Aufstandes der Herero im heutigen Namibia - in Deutschland nahezu verschwiegen wurde ${ }^{4}$ (dazu auch Campt 2004; El-Tayeb 2001). Ursula Wachendorfer (1998) schrieb den ersten Text zum Weißsein* und weitere Werke regten das Nachdenken darüber an (Fuchs und Habinger 1996; Rommelspacher 1995; Mamozai 1982).

Bis zum Ende des 20. Jahrhunderts vermochten die einzelnen Studien jedoch keine breitere Debatte über Whiteness anzuregen. Erst Anfang des 21. Jahrhunderts wurde „Critical Whiteness“ zum Thema im deutschsprachigen Raum und Mitte der 2000er Jahre zu einem eigenständigen Forschungsfeld. Nach und nach erschienen einzelne Monographien - zumeist Dissertationen, aber auch interdisziplinär angelegte Sammelbände (Tißberger et al. 2006; Eggers et al. 2005) und Forschungsübersichten (Röggla 2012; Amesberger und Halbmayr 2008), sowie auch konkrete Ausarbeitungen im Zusammenhang mit Diversity und Intersektionalität (Tißberger 2019, 2017, 2016).

\section{Soziale Arbeit als weißer* Raum - nachgeforscht}

Rassistisch markierte Menschen stellen eine signifikante Größe unter den Adressat*innen der deutschsprachigen Sozialen Arbeit dar, denn sie werden einerseits in allen Bereichen des gesellschaftlichen Lebens diskriminiert und geraten dadurch

\footnotetext{
3 Kritisch zum Begriff der Privilegien in den Critical Whiteness-Diskursen: Leonardo (2005).

4 Ausnahmen sind Zeller (2000) und Zimmerer und Zeller (2003).
} 
in prekäre Lebenslagen, andererseits werden sie von der weißen* Dominanzkultur durch die koloniale Episteme (Quijano 2016; Mignolo 2012) per se als „,sozial bearbeitungsbedürftig“ konstruiert. Sie engagieren sich nicht zuletzt aufgrund ihrer Diskriminierungserfahrung häufig dafür, Teil der Professionellen der Sozialen Arbeit in Praxis, Forschung und Lehre zu werden, doch auch dort stoßen sie auf ausgrenzende Strukturen. Die weißen* Mehrheitsangehörigen in der Sozialen Arbeit sind sich dessen selten bewusst. Wir haben deshalb ein qualitatives Lehrforschungsprojekt zur Bedeutung von Whiteness in der Sozialen Arbeit in Österreich am Masterstudiengang Soziale Arbeit der Fachhochschule Oberösterreich in Linz durchgeführt (Oktober 2014-Januar 2016) ${ }^{5}$. Hierzu wurden verschiedene Bereiche einbezogen. Wir führten mit sechs Sozialarbeiter*innen, sechs Adressat*innen, drei Lehrenden und sechs Studierenden der Sozialen Arbeit narrative Interviews (Rosenthal 2000, 1995). Außerdem wurde eine Diskursanalyse (Parker 2013; Jäger 2006, 1993) von in der Öffentlichkeit zugänglichen Leitbildern, Jahresberichten, etc. von elf Organisationen der Sozialen Arbeit in (Ober-)Österreich durchgeführt. Bei der Auswertung wurde der Fokus auf die Bedeutung und Funktion von Whiteness, die Intersektion von Rassismus und Gender sowie die Interdependenzen zwischen personaler, symbolisch-repräsentativer und institutioneller Ebene gerichtet (vgl. Winker und Degele 2009). Hier stehen die Sozialarbeiter*innen im Fokus, bei denen sich unser Forschungsinteresse auf die Frage richtete, ob sie sich ihres (mehrheitlichen) Weißseins* als Position im Geflecht der gesellschaftlichen Machtverhältnisse und deren rassistischer Matrix bewusst sind.

Die erzählgenerierende Eingangsfrage für die Interviews stellte eine Herausforderung dar, denn die Frage nach dem eigenen Weißsein* löst bei den meisten Mitgliedern der deutschsprachigen Dominanzkulturen nicht, wie es die Methode will, Lust zum Erzählen, sondern Irritation aus (Tißberger 2017). Das „Unbehagen in der weißen* Kultur“ (Tißberger 2013) stellt sich ein. Also formulierten wir unsere Erzählaufforderung unverfänglicher:

Ich möchte Sie bitten, mir über Ihre Arbeit vor dem Hintergrund der Machtverhältnisse des Rassismus und der Geschlechterverhältnisse und ihrer eigenen Position darin zu erzählen. Sie können anfangen, wo Sie wollen und sich so viel Zeit nehmen, wie Sie möchten. Mich interessiert alles. Ich werde Sie nicht unterbrechen, sondern mir Notizen machen und später nachfragen.

Bei der Kontaktaufnahme schilderten wir den Interviewpartner*innen allerdings detailliert unser Forschungsinteresse und so wurden auch Whiteness und das Weißsein* thematisiert.

Gabriele Rosenthals (2000) narrativer Interviewführung zufolge wird nach der erzählgenerierenden Eingangsfrage und der darauffolgenden Stegreiferzählung zunächst erzählimmanent entlang der angefertigten Notizen nachgefragt. Es werden also die Themen vertieft, die bereits in der Stegreiferzählung auftauchen. Erst am Ende werden externe Fragen zu den Themen gestellt, die von den Interviewpartner*innen ausgelassen, aber für das Forschungsinteresse wichtig sind. Auf diese Weise lässt sich als Forscher*in erfahren, wie die Interviewten Themen innerhalb

\footnotetext{
5 Der studentische Forschungsbericht ist als Buchbeitrag erschienen: Tißberger et al. 2016.
} 
eines Feldes priorisieren. Für uns war beispielsweise besonders interessant zu erfahren, ob und an welcher Stelle das eigene Weißsein* zur Sprache kommt, welche Beispiele für Rassismus gegeben und in welcher Reihenfolge sie erzählt werden und ob Intersektionen wahrgenommen werden.

Drei der Interviewpartner*innen arbeiteten in Einrichtungen, die explizit migrantisch markiertes Klientel adressieren, die anderen drei in Einrichtungen, bei denen das nicht der Fall ist, in denen sich aber dennoch ein hoher Anteil an migrantisch markierten Menschen unter den Adressat*innen befinden. Zwei der Interviewpartner*innen arbeiteten in einer Migrant*innen-Selbstorganisation, alle anderen bei den großen Trägern.

Die einzige Person, die von alleine auf ihr Weißsein* zu sprechen kam, war selbst migrantisch markiert und arbeitete in einer der Migrant*innen-Selbstorganisationen. In dieser Organisation sind Postkoloniale Theorie, Dekolonialität, Feministische- und Queer Theory, Intersektionalität, Critical Whiteness und andere kritische Ansätze handlungsleitend. Das macht sie zu einer Ausnahme in der Sozialarbeitslandschaft (Ober-)Österreichs. Alle anderen Interviewpartner*innen arbeiteten in ihren Narrationen zwar die Frage nach Machtverhältnissen, Rassismus, Gender und auch anderen mit Rassismus intersektional verknüpften Machtvershältnissen wie beispielsweise Klasse ab, thematisierten ihre eigene Position innerhalb der rassistischen Gesellschaftsmatrix jedoch nicht. Sie wichen sogar den dies thematisierenden Nachfragen aus. Eine der interviewten Personen, die in ihrer Narration mehr als eine Stunde lang den Rassismus unter den Nutzer*innen der Einrichtung, der Polizei, den Behörden sowie die Intersektionen von Rassismus und Gender thematisiert hatte, ohne ihre eigene Rolle dabei zu reflektieren, wurde gegen Ende des Interviews direkt gefragt:

Also so, wenn man jetzt im Kontext von Rassismus denkt, dann sind wir als weiße* Sozialarbeitende, die halt in Österreich geboren sind, ja schon in einer Machtposition jetzt. Und wie erlebst du das in deiner Arbeit jetzt, also diese Machtposition in dem Machtverhältnis?

Sie reagierte irritiert mit der Frage:

\section{1 ,Was meinst du jetzt mit weißem* Sozialarbeiter?“6}

Mitglieder der weißen* Mehrheitsgesellschaften in den deutschsprachigen Ländern erleben sich, wie oben geschildert, generell nicht als weiße* Subjekte, sondern denken sich vom normativen Standpunkt aus, der für sie nicht erläuterungsbedürftig ist. Auch diejenigen, die sich als „Antirassist*innen“ definieren, denken beim Thema Rassismus an „Täter*innen“ und Strukturen, die sich jenseits ihres eigenen Standpunktes als liberales, humanistisches Subjekt einer demokratischen Gesellschaft, nämlich an dessen Rand, befinden. Sozialarbeitenden als Professionelle einer Menschenrechtsprofession fällt es jedoch besonders schwer, sich vorzustellen, dass sie schon allein durch ihr Weißsein* Teil des Rassismus sind, denn sie wähnen sich per se auf der „guten Seite“. Dass die gesamte Gesellschaft rassistisch strukturiert ist, nehmen die Sozialarbeiter*innen mehrheitlich nicht wahr. 
Alle unsere Interviewpartner*innen berichteten detailliert über den Rassismus, den ihre Adressat*innen auf Ämtern, Behörden, bei der Polizei, im Gesundheitswesen etc. erfahren. ${ }^{6}$ Den Raum der Sozialen Arbeit verorteten sie jedoch jenseits ihrer Landkarte des Rassismus. Sie wird als „,sicherer Ort“ für „Migrant*innen“ wahrgenommen, wo sie unterstützt und geschützt werden, auch vor dem Rassismus „draußen“ in der Gesellschaft. Die Institution der Sozialen Arbeit immunisiert also gewissermaßen gegen die Erkenntnis, dass auch wohlmeinende, „gute“ Menschen, die sich zur Aufgabe gemacht haben, anderen Menschen zu helfen, im Kontext des Rassismus Teil des Problems und nicht der Lösung sein können.

Zum Thema Rassismus fällt zwei der Interviewpartner*innen zuerste der vermeintliche Rassismus unter den Adressat*innen ein. Ein Interviewpartner, der in der Geflüchtetenhilfe arbeitet, formuliert es in seiner Stegreiferzählung so:

Ja, im Bezug auf Machtverhältnisse und Rassismus, was kann ich da dazu sagen? Also, was mir schon auffällt ist, also Rassismus herrscht viel unter den Klienten selber, also das merkt man schon sehr. Wenn sie mit mir reden, sagen sie mir, wie toll es ist, dass sie in Europa sind, und dass es auch egal ist, welche Religion und welches Geschlecht und was du halt machst, dass das alles egal ist. Aber untereinander, zum Beispiel Syrer mit Afghanen, Afghanen mit Pakistani, gibt es eigentlich immer relativ viele Vorbehalte, muss man sagen. Komm ich dann oft so in die Lage, wo ich dann quasi auch vermittelnd bin. [...] Es gibt Einzelfälle, wo dann ein Klient sagt: naja, na gut, also. Ein christlicher Iraker kann ich mich erinnern. [...] Der hat sich halt aufgeregt, dass im Haus so viele Muslime sind. Er hat gesagt, nein, er will in ein Haus, wo nur Christen gibt. Ist ein Ding der Unmöglichkeit, weil es das eigentlich nicht gibt. Ahm sonst, was kann man sonst zu Rassismus sonst so sagen. Gibt es eigentlich nicht viel Probleme, muss ich sagen.

An späterer Stelle des Interviews kommt er der Bitte, das Thema zu vertiefen, wie folgt nach:

Jetzt hab ich ein Haus, da wo relativ viel Syrer und Araber aah und Afghanen sind. Da herrscht dann schon immer wieder das Bild von Syrern öfters, es wird geglaubt, die Afghanen sind ja alles die Bauern und denen macht der Dreck ja eh nichts aus und so was.

Es folgt eine lange Sequenz, in der er erläutert, wie er in die Rolle des Vermittlers und Mediators kommt (,,also da ist immer wieder recht viel Aufklärung von Nöten“). Er muss also diese Klient*innen zum Thema Gleichberechtigung aufklären. Wenn die Syrer etwa fordern:

Gebt uns Asyl, weil die da, denen ist es eh nicht [...] naja nur weil der ein Afghane ist, oder was weiß ich, dann ist der nicht so wichtig, ob der in seinem Heimatland bedroht wird, weil für die herrscht oft wirklich auch so die Ansicht,

\footnotetext{
6 vgl. auch Wagner (2017).
} 
die sind ja wirklich? (Ob die jetzt sterben oder nicht), mein Gott. Aber wir sind die wichtigen, uns müsst ihr retten.

Eine Interviewpartnerin, die in der Wohnungslosenhilfe arbeitet, formuliert es so:

Rassismus ist auch immer wieder ein Thema, vor allem [...] zwischen den Klienten, also unter den Klienten und Klientinnen spielt das eine große Rolle. Wo es auch viel Diskriminierungen gibt und wo man immer wieder im Team überlegen, wie wir auf so etwas regieren können, wie wir damit umgehen (2). Ich denke mir, dass wir als Team schon auch immer viel reflektieren über das.

Angesichts dessen, wie stark rassistisch markierte Menschen - zumal Geflüchtete - in Österreich sämtlichen Formen des Rassismus durch die weiße* Mehrheitsgesellschaft ausgeliefert sind, ist es bemerkenswert, wenn auf die Frage nach der Bedeutung von Rassismus und Machtverhältnissen in der Sozialen Arbeit diese Interviewpartner*innen, die (ausschließlich) mit rassistisch markierten Adressat*innen arbeiten, die „Opfer“ zu Täter*innen machen. Ich habe diese Reaktion auch häufig in Lehrveranstaltungen und anderen Kontexten der Sozialen Arbeit erlebt; beim Thema Rassismus wird sofort über den der Adressat*innen gesprochen. Aus den Interviews beider Sozialarbeiter*innen geht hervor, dass ihnen bewusst ist, dass Migrant*innen in Österreich generell rassistischer Diskriminierung ausgesetzt sind und sie nennen zahlreiche Beispiele, wo sie durch die Zusammenarbeit mit ihren rassistisch markierten Adressat*innen diesen Rassismus direkt miterleben. Auch wenn sie glauben, als Sozialarbeiter*innen nichts mit diesem Rassismus zu tun zu haben, sondern zu den ,guten“ Österreicher*innen zu gehören, ist es überraschend, dass sie das diskriminierende Verhalten zwischen Geflüchteten ins Zentrum ihrer Diskussion über Rassismus stellen. Dieser Abwehrmechanismus der Reaktionsbildung schützt sie davor, über den Rassismus der Österreicher*innen und vor allem ihren eigenen Rassismus nachdenken zu müssen.

Wie die narrative Konstruktion des ersten Interviewauszugs zeigt, werden die Österreicher*innen als Europäer*innen und damit als Weiße* von den Asylwerbenden für ihre vermeintlich fortschrittliche, diskriminierungsfreie Kultur bewundert. Die Klient*innen sind nach Europa geflohen, weil sie in ihren Herkunftsländern verfolgt werden oder Krieg herrscht und dabei spielen, wie die narrative Konstruktion nahelegt, religiöse Motive eine zentrale Rolle. Der Klassismus, der von Adressat*innen geäußert wird und der die ,afghanischen Bauern“ gar als lebensunwürdig herabwertet, verweist überdies auf die vermeintliche ,zivilisatorische Rückständigkeit“, die den Geflüchteten zugeschrieben wird. So wird jegliche Verantwortung für die derzeitigen Kriege in der ,,arabischen Welt“ auf die „Kultur“ der betroffenen Länder verschoben. Die (neo-)kolonialen Beziehungen zwischen „dem Westen“ und den muslimischen Ländern, in denen westliche Mächte religiöse und ethnische Gruppen nach dem Prinzip des Teilens und Herrschens seit Jahrhunderten gegeneinander ausspielen, die Abhängigkeit des Kapitalismus von den Öl-produzierenden Ländern, die geostrategischen und wirtschaftlichen Interessen, die ,der Westen“ rücksichtslos und zum Teil gewaltförmig durchgesetzt hat, wodurch die Regierungen der muslimischen Länder korrumpiert wurden, sind kein Thema in den Narrationen der weißen*, österreichischen Sozialarbeiter*innen über Geflüchtete. Die koloniale Episteme wird 
von den Interviewpartner*innen reproduziert, ohne dass ihnen das bewusst wird. Die Geflüchteten sind die Rassist*innen, die weißen*, österreichischen Sozialarbeiter*innen vertreten dagegen die Menschenrechte.

Eine weitere Strategie zur Subversion der Verhältnisse und zur Immunisierung der Sozialen Arbeit gegen die Erkenntnis ihrer Teilhabe an der Kolonialität der Macht ist also das professionelle Selbstverständnis des Vermittelns, Aufklärens, Schützens, Helfens und Reflektierens - allesamt Fähigkeiten, die in den Narrationen ausschließlich zur sozialarbeiterischen Selbstbeschreibung, nicht aber für die Adressat*innen benutzt werden. Dabei vermitteln migrantisch markierte Adressat*innen ständig zwischen Sozialarbeiter*innen oder Mitarbeiter*innen von Behörden, der Polizei oder dem Arbeitsamt und anderen Migrant*innen, die noch nicht Deutsch sprechen. Sie klären über Sachverhalte auf und helfen Adressat*innen wie auch Sozialarbeiter*innen in Einrichtungen. Sie sind häufig gezwungen zu reflektieren, da sie durch die Migration mit sehr unterschiedlichen Lebensbedingungen konfrontiert sind, sich anpassen und ihre Gewohnheiten ändern müssen.

Wenn nicht das Verhalten von Adressat*innen als erstes Beispiel für Rassismus genannt wird, sind es andere Institutionen: Behörden, Ämter, das Magistrat, andere Einrichtungen der Sozialen Arbeit, vor allem aber die Polizei. Eine Mitarbeiterin in einem Gewaltschutzzentrum kommt zum ersten Mal im Interview wie folgt auf Rassismus zu sprechen:

Und ahm, ja was Rassismus angeht, gibt es schon natürlich auch immer wieder (3) mehr oder weniger große Auffälligkeiten, ahm also wie, wie natürlich mit Leute umgegangen wird, die, sage ich einfach mal, andere Hautfarbe haben, eine gewisse ethnische Zugehörigkeit haben, sei es jetzt durch Polizeibehörden, ja nur als Beispiel. [...] Ja, es ist natürlich, ich sage einmal für Migranten, ist oft Zugang schwieriger schon einmal alleine schon zu Behörden. Das kann sprachlichen Hintergrund haben, wenn ich nicht perfekte Sprache sprich, also entweder gar nicht spreche oder nicht sehr gut spreche, ahm, beziehungsweise da rede ich jetzt auch von Deutsch und Dialekt.

Rassismuserfahrungen, die Adressat*innen außerhalb der Einrichtung der Interviewpartner*innen, etwa bei anderen Einrichtungen der Sozialen Arbeit, vor allem aber auf Ämtern, bei Behörden und der Polizei machen, werden von allen Interviewpartner*innen mehrfach und über die komplette Zeitspanne ihrer Interviews hinweg geschildert. Bei all diesen Narrationen wird die Normativität von Whiteness deutlich und auch, wie diese Norm ,versteckt“ wird. Die Formulierung, Menschen, die eine ,andere Hautfarbe“ haben, adressiert konkret die Rasse-Konstruktion und zwar als Abweichung, während die Norm, bzw. das Ideal des Weißseins*, das durch diese Formulierung performativ hervorgebracht wird, sorgfältig versteckt bleibt. Auch die Begründungsfigur, dass jemand ,eine gewisse ethnische Zugehörigkeit“" hat und deshalb eine bestimmte Behandlung erfährt, beruht auf der Grundlage, dass die Norm entweder nicht ethnisch markiert ist oder diese Ethnizität keiner Erwähnung bedarf. Schließlich gibt es in dieser Erzählung die Sprache, die man beherrscht oder eben nicht. Gerade, indem sie nicht benannt wird, wird die deutsche Sprache normativ. Dass Migrant*innen (im Gegensatz zu vielen weißen* Österreicher*innen) häufig neben ihrer Erstsprache eine Reihe anderer Sprachen beherrschen, geht nicht in den 
Diskurs ein. Was zählt, ist nur die Sprache, an deren Beherrschung letztendlich Menschenrechte gebunden werden. Wir hörten in vielen Interviews, dass Adressat*innen, die ,nicht sehr gut“ Deutsch sprechen, bei Behörden wichtige Dokumente nicht ausgehändigt, Informationen vorenthalten und Bewilligungen verwehrt werden. Auch die für Außenseiter*innen schwer verständlichen österreichischen Dialekte werden häufig im Dienst von Diskriminierung eingesetzt.

Eine Sozialarbeiterin in der Bewährungshilfe spricht fast eine Stunde, ehe der Begriff Rassismus fällt. Sie bezieht rassistisches Verhalten dann allerdings umgehend auch auf ihr eigenes Team:

So, was haben wir jetzt, Rassismus noch gar nicht. Rassismen, naja was wir uns sicher zu wenig anschauen, ähm, was in anderen Bereichen hier und da Thema wird, ist dieses Ethnic Profiling, dass man sagt, das ist ein Türke und er ist deswegen so. Ähm bemerke ich manches Mal in Fallbesprechungen, dass es auch so Bemerkungen gibt, wo wir auch sagen, wenn man im Fall, wenn man in den Fallbesprechungen so sind, da haben wir ein offenes Klima, dass es dann auch ähm Kollegen, Kolleginnen gibt, die einen darauf hinweisen oder man selber irgendwie sagt, he, bitte jetzt das. Ähm aber das ist (1) habe ich durchaus auch schon im Kollegenkreis gehört, so eben Russe, Tschetschene, der hat sich so irgendwie umgeschaut und dann ist es so ausgelegt worden, also immer, wenn er wo hingekommen ist, naja üblicherweise stehlen die.

Sie räumt ein, dass auch Sozialarbeiter*innen rassistische Konstruktionen reproduzieren, Rassismus bleibt jedoch auf der Ebene des Individuums. Die strukturelle und epistemische Dimension, etwa die Tatsache, dass noch so kriminelle weiße* Österreicher*innen nie im Raster des „Ethnic Profiling“ festgehalten werden, bleibt dethematisiert. Schließlich wird auch in dieser Formulierung Rassismus in der Sprache versteckt. Der offizielle Begriff lautet „Racial Profiling“ und ist im Bereich der Polizeiarbeit verortet. Der hässliche Rasse*-Begriff steckt also darin und verweist auf den Rassismus der Gesellschaft. Der Begriff beschreibt zudem eine systemische, symbolisch-strukturelle, epistemische Dimension des Rassismus; er verweist direkt auf die rassistische Matrix der Gesellschaft, deren sich die Polizei bedient. Die Interviewpartnerin verschiebt also einen Begriff von der strukturellen Ebene auf die individuelle (einzelne Kolleg*innen im Team äußern sich ethnisierend) und versteckt Rasse* in Ethnizität. Racial Profiling basiert auf der rassistischen Konstruktion, dass die äußere Erscheinung von Menschen Hinweise darauf gibt, wie sie denken, fühlen und handeln. So wird beispielsweise eine Hautfarbe mit Kriminalität verknüpft. Die Ontologisierung von Kriminalität benutzt also den Körper als Vehikel. Ethnizität dagegen wird im Bereich der Kultur verortet, sie ist also eine flexible Kategorie. Kulturelles Verhalten wird erlernt, kann aber auch wieder verlernt werden. Eine Hautfarbe kann dagegen nicht abgelegt werden.

Eine dem oben Geschilderten vergleichbare Selbstkritik taucht in den Interviews nicht oft in Bezug auf das eigene Team auf. Viel häufiger sind die Erzählungen, in denen sich die Sozialarbeiter*innen als Opfer anderer Institutionen, etwa der Asylbehörde, vor allem aber als Opfer der Politik und sogar ihrer Klient*innen erleben. In der Vertretung der Interessen ihrer Klient*innen als Asylwerbende, Jobsuchende etc. geraten sie schnell in den berühmten Widerspruch des Tripelmandats der Sozia- 
len Arbeit (Staub-Bernasconi 2018). Mehrere unserer Interviewpartner*innen hoben hervor (und dies in den Sequenzen, in denen sie gebeten wurden, über ihre dominante Rolle im gesellschaftlichen Machtverhältnis als weiße*, relativ gebildete und wohlhabende Professionelle zu sprechen), dass sie ganz und gar nicht auf der Seite der Mächtigen stehen. Vielmehr kämpfen sie für die Rechte ihrer Adressat*innen und bekommen als deren Vertreter*innen den Rassismus der weißen* österreichischen Beamt*innen bei den Behörden zu spüren.

Die Adressat*innen verstünden dies nur leider nicht immer, was, so wird nahegelegt, mit ihrem Bildungs- und Informationsmangel zu tun habe; daher betrachteten sie die Sozialarbeiter*innen genauso wie die Mitarbeiter*innen der Asylbehörde als ihre Feinde. Ein Interviewpartner empört sich über eine dieser Situationen: „Ich habe ja nicht die Macht. Das ist ja oft eine fälschliche Macht, die mir dann zugeschrieben wird" und erläutert dann, wie schwierig es ist, den Adressat*innen etwa zu erklären, dass nicht er, sondern die Asylbehörde über ihren Antrag entscheidet oder er die Mindestsicherung nicht auszahlen darf, weil die Adressat*innen bestimmte Voraussetzungen nicht erfüllen. Da müsse viel „Vertrauensarbeit geleistet“ werden, sagt er. Tatsächlich ist es für die Soziale Arbeit immer eine Herausforderung, ihre drei Mandate angemessen zu erfüllen. Die Wahrnehmung, dass es lediglich ein Missverständnis auf Seiten der rassistisch markierten Adressat*innen ist, dass Sozialarbeiter*innen auf der Seite der Mächtigen stünden, ist jedoch falsch, wie im Folgenden weiter gezeigt werden soll.

\subsection{Weiße* Kompliz*innenschaft}

Mehrheitlich erzählen unsere Interviewpartner*innen unaufgefordert von Situationen, in denen sie ihre rassistisch markierten Adressat*innen auf ein Amt, eine Behörde, zur Polizei oder zu einer Ärztin begleiten, und ihnen dort dann Rechte und Dienstleistungen gewährt werden, die ihnen zuvor, ohne Begleitung, verwehrt wurden. Eines dieser Beispiele ist das einer Sozialarbeiterin, die ihre Klientin zur Polizei begleitet, um Anzeige wegen eines Nachbarschaftskonflikts zu erstatten:

Weil sie tatsächlich verletzt worden ist. Und ich war wirklich total froh, dass ich mitgegangen bin zur Anzeige, weil ich habe gesehen, auch durch meine Anwesenheit hat sich einiges abgemildert. Ja, aber ich glaube, das wäre total in die Hose gegangen, wenn ich nicht dabei gewesen wäre. Die hätte vielleicht gar keine Anzeige machen können, das wäre nicht einmal aufgenommen worden. Wo man merkt, so dieses Ernstnehmen ist nicht so da. Das war noch dazu eine, eine Frau, sage ich mal, die so vom Auftreten her ein bisschen traditioneller gewirkt hat, sag ich jetzt mal, aus Westafrika, wo von der Sprache, der Ausdrucksweise, von, von den Gebärden oder was halt, ist halt nicht ganz so, wie es eine Österreicherin machen würde. Und da wird ganz viel belächelt und ins Lächerliche gezogen [...] dass die Leute nicht so ernst genommen werden, sie nicht als so glaubwürdig angenommen werden und vielleicht nicht so genau ermittelt wird (3), schneller was unterstellt wird (5), dass sich die Leute viel mehr rechtfertigen müssen für Dinge, die, für die es eigentlich keinen Rechtfertigungsgrund gäbe, ja (18). 
Nachgefragt, wie sie sich in solchen Situationen fühlt, antwortet sie: „Ein kleines bisschen befriedigend, dass ich in dem konkreten Fall helfen habe können (3). Aber eigentlich sollte das nicht nötig sein. Das ist eigentlich das vorherrschende Gefühl dabei.“

Was ihre Anwesenheit auf der Wache genau bewirkt hat, beschreibt sie nicht. Sie setzt es als selbstverständlich voraus, dass ihre Interviewerin und alle Mehrheitsangehörigen genau wissen, was sie meint. Die „Differenz“ der Klient*in wird beschrieben, aber von wo aus diese definiert wird, bleibt seltsam unbeschrieben. Im Beisein einer weißen* Person, zumal einer Vertreterin einer Einrichtung der Mehrheitsgesellschaft, können die Polizist*innen ihren Rassismus scheinbar nicht so ungehemmt ausleben, wie wenn eine rassistisch markierte Person alleine vor ihnen steht. Der Sozialarbeiterin fällt die weiße* Kompliz*innenschaft, an der sie beteiligt wird, jedoch nicht auf. Es sollte nicht so sein, wie sie sagt, aber was da eigentlich genau passiert, analysiert sie nicht.

Wenn sie ihre Adressat*innen zu Behörden oder zu Ärzt*innen begleiten, erleben weiße* Sozialarbeiter*innen oft, dass die Ärztin nicht mit den rassistisch markierten Adressat*innen, sondern mit der Sozialarbeiter*in spricht, auch wenn die Adressat*innen gut Deutsch sprechen. Aber auch in diesen Sequenzen der Narrationen umschiffen die Interviewpartner*innen versiert das symbolische Kapital von Whiteness, das für dieses Verhalten verantwortlich ist. Sie benennen ihre Professionalität, Sprachbarrieren oder andere Aspekte als Grund dafür, dass Weiße* über Nicht-Weiße* sprechen, statt mit ihnen, nicht aber das Weißsein* und die Kompliz*innenschaft, die sich unter Weißen* herstellt und die dafür sorgt, dass plötzlich Dienstleistungen möglich und Ressourcen frei gemacht werden, die Nicht-Weißen* illegitimerweise vorenthalten werden.

Ein Sozialarbeiter im Bereich Asyl erzählt von Schwierigkeiten, die es immer wieder in Quartieren gibt, die von Privatpersonen betrieben werden. Als Betreuer wird er von den Klient*innen beispielsweise gebeten, den Quartiersbetreiber dazu zu bewegen, endlich die Waschmaschine zu reparieren. Im Gespräch sieht der Betreiber den Sozialarbeiter nicht als den Anwalt der Bewohner*innen, sondern glaubt ihn auf ,seiner“ Seite, denn er erklärt ihm, dass die Bewohner*innen ,,ja alles absichtlich hinmachen und dass das einfach solche Tiere sind, die nicht auf nichts Acht geben können.“ Er rechnet also mit dem „Pakt unter Weißen*“ und zählt auf den rassistischen Konsens.

Wenn Sozialarbeiter*innen solche Situationen erleben, sehen sie Individuen oder bestimmte Milieus für Rassismus verantwortlich. Dass die Gesellschaft durch eine rassistische Matrix strukturiert ist, in der Weißsein* immer mit Macht einhergeht, blenden die meisten unserer Interviewpartner*innen aktiv aus. Zumindest soll es auch Räume geben, die von Rassismus frei sind und die sehen sie in ihrer Praxis der Sozialen Arbeit. Auf das beharrliche Nachfragen nach dem Nexus von Whiteness und Macht in der Sozialen Arbeit antwortet ein Interviewpartner:

[...] weil ich es überhaupt nicht so empfinde. Dass weiße* Sozialarbeiter (anders), das empfinde ich in der Arbeit einfach nicht so. Wir haben viele Migranten, die auch als Sozialarbeiter, aber da gibt es, glaube ich, zum Beispiel auch keine Machtunterschied. Also ob das jetzt weiße* Sozialarbeiter oder (.) Sozi- 
alarbeiter ist oder Dolmetscher haben wir viele, die, da würde ich jetzt nicht sagen, dass es da irgendeinen Unterschied gibt bei Macht. Eben eher sogar, Migranten haben sogar um einiges besser würde ich sagen, weil sie sich oft mit der Sprache viel leichter tun. Und es wird auch glaube ich bei uns immer geschaut, wenn neue Mitarbeiter eingestellt werden, wird das sogar, ist das was Gutes. Also wenn wirklich ein Migrant ist, der jetzt schon da ist, zweisprachig ist, vielleicht eben noch Sozialarbeiter ist, oder auch nicht, ist glaube ich auch nicht immer, dass das ein irrsinniger Vorteil, von uns weißen* Sozialarbeitern sogar sehr geschätzt wird, weil wir sie es eben auch als Dolmetscher heranziehen können.

Auch hier können wir eine Subversion beobachten. Zur Untermauerung seines Arguments, dass es keine rassistischen Machtverhältnisse in seiner Organisation gibt, verweist der Sozialarbeiter auf die mehrsprachigen Mitarbeiter*innen, die vor allem als Dolmetscher*innen instrumentalisiert und auf die Arbeit mit Migrant*innen reduziert werden. Dass neuerdings eine Antidiskriminierungs-Personalpolitik betrieben wird, die mehrsprachige Bewerber*innen für offene Stellen bevorzugt (und das häufig nur in Einrichtungen, deren Zielgruppe Migrant*innen sind), interpretiert er sogar gewissermaßen als Diskriminierung von Weißen* - Migrant*innen haben es „sogar um einiges besser.“ Ausgeblendet wird, dass seit langem und immer noch, viele Einrichtungen der Sozialen Arbeit (in Österreich) kaum migrantisch und rassistisch markierte Mitarbeiter*innen beschäftig(t)en. Der omnipräsente Rassismus wird ausgeblendet. Erst wenn diese strukturelle Diskriminierung als Norm durch eine affirmative Personalpolitik destabilisiert wird, gerät das Thema Diskriminierung ins Blickfeld und nicht selten erleben sich die, welche die längste Zeit massiv privilegiert wurden, plötzlich als Opfer von Diskriminierung.

Schließlich stellt sich die Frage, wie diese Verhältnisse verändert werden können. Der letzte Abschnitt kann lediglich zwei wesentliche Aspekte dazu skizzieren. Die angemessene Beantwortung der Frage bräuchte den Raum eines eigenen Aufsatzes. Auch im Rahmen des Lehrforschungsprojektes, das hier zitiert wurde, haben wir es einem Folge-Projekt überlassen, das Thema auszuarbeiten (Tißberger et al. 2017).

\section{Critical Whiteness als Praxis hegemonialer Selbstreflexion in der Sozialen Arbeit}

Wenn die Diagnose nicht stimmt, wird auch das Heilmittel nicht wirken. Eine Auseinandersetzung mit Rassismus und der Versuch, ihm möglichst wenig Raum zu geben, können in der Sozialen Arbeit nur gelingen, wenn Rassismus als gesamtgesellschaftliches, strukturelles Phänomen verstanden wird, in das alle Subjekte mehr oder weniger verstrickt sind. Wie ich gezeigt zu haben hoffe, gibt es keinen Raum jenseits des Rassismus in einer Gesellschaft, die rassistisch strukturiert ist. Auch wenn die Soziale Arbeit ihr Mandat als Menschenrechtsprofession gewissenhaft umsetzen und weder Rassismus noch andere Diskriminierungen akzeptieren will, leben wir in einer Gesellschaft, die durch eine über 500 Jahre alte Geschichte des Kolonialismus und Rassismus geprägt ist. Die koloniale Episteme mit ihrem rassistischen 
Wissensarchiv ist in unserem Bewusstsein und Unbewussten sedimentiert. Mit Kolumbus' vermeintlicher „Entdeckung“ Amerikas 1492 wird die Moderne eingeläutet, deren konstitutive Mitbedingung die Kolonialität ist. Rasse* wiederum fungiert als Naturalisierung der kolonialen Macht- und Herrschaftsverhältnisse, die mit diesem epochalen Ereignis initiiert wurden und bis in die heutigen (Post-)Migrationsgesellschaften hineinwirken. Es gilt also, epistemischen Ungehorsam (Mignolo 2012) zu leisten. Gerade die als weiß* positionierten Mitglieder unserer Gesellschaften müssen sich ihres rassistischen Wissensarchivs bewusst werden und verstehen, wie es ihr Denken, Fühlen, Sprechen und Handeln beeinflusst. Dieses rassistische Wissen sollte gegen Wissen über Rassismus ausgetauscht werden. Wir müssen also ,lernen, um das koloniale Wissen zu verlernen“ (Mignolo 2012, S. 41). Das geschieht nicht in einem Wochenend-Seminar. Es braucht Geduld. Es ist eine lebenslange Aufgabe.

Protagonist*innen in dieser kolonialen Episteme sind wir nicht nur wegen unseres rassistischen Wissens, sondern wegen der konstitutiven Bedeutung von Rasse* für die Kolonialität der Macht auch durch unsere Körper und das symbolische Kapital, das mit dem Weißsein* einhergeht. Weiße* müssen verstehen lernen, wohin sie ihr Weißsein* gehen lässt und wohin nicht, was es für andere Menschen bedeutet und wie es Beziehungen zu Nicht-Weißen* strukturiert. Erst wenn wir begreifen, welche Bedeutung Whiteness für die Hegemonie in deutschsprachigen Gesellschaften hat, lässt sich Soziale Arbeit als Menschenrechtsprofession praktizieren, ohne vom Unbewussten des Rassismus korrumpiert zu werden. Denn der Rassismus, den Sozialarbeiter*innen gewöhnlich in deutschsprachigen Gesellschaften erkennen können - der ihnen also bewusst ist - stellt nur die Spitze des Eisbergs dar. Wie ich in den empirischen Beispielen zu zeigen versuchte, sind weiße* Sozialarbeiter*innen häufig gerade einmal Zaungäste in der Arena des Kampfes gegen Rassismus. Sie sollten Protagonist*innen darin werden. Wenn sie keine Kompliz*innen der kolonialen Macht sein wollen, muss Weißsein* reflektiert und diese Kompliz*innenschaft durchkreuzt werden. Der rassistische Pakt ist aufzukündigen und zwar auf allen Ebenen: der personalen, der institutionellen und der symbolisch-repräsentativen. Critical Whiteness als hegemoniale Selbstreflexion zu praktizieren bedeutet, sich konstruktiv zu verunsichern; Verunsicherung als Methode anzuwenden (Tißberger 2005), oder wie bell hooks (1994b) sagen würde: ,to become comfortable with being uncomfortable“.

Open Access Dieser Artikel wird unter der Creative Commons Namensnennung 4.0 International Lizenz veröffentlicht, welche die Nutzung, Vervielfältigung, Bearbeitung, Verbreitung und Wiedergabe in jeglichem Medium und Format erlaubt, sofern Sie den/die ursprünglichen Autor(en) und die Quelle ordnungsgemäß nennen, einen Link zur Creative Commons Lizenz beifügen und angeben, ob Änderungen vorgenommen wurden.

Die in diesem Artikel enthaltenen Bilder und sonstiges Drittmaterial unterliegen ebenfalls der genannten Creative Commons Lizenz, sofern sich aus der Abbildungslegende nichts anderes ergibt. Sofern das betreffende Material nicht unter der genannten Creative Commons Lizenz steht und die betreffende Handlung nicht nach gesetzlichen Vorschriften erlaubt ist, ist für die oben aufgeführten Weiterverwendungen des Materials die Einwilligung des jeweiligen Rechteinhabers einzuholen.

Weitere Details zur Lizenz entnehmen Sie bitte der Lizenzinformation auf http://creativecommons.org/ licenses/by/4.0/deed.de. 


\section{Literatur}

Amesberger, H., \& Halbmayr, B. (2008). Das Privileg der Unsichtbarkeit. Rassismus unter dem Blickwinkel von Weißsein und Dominanzkultur. Wien: Braumüller.

Campt, T. (2004). Other Germans: Black Germans and the politics of race, gender, and memory in the Third Reich. Ann Arbor: University of Michigan Press.

Castro Varela, M. (2012). Interkulturelle Kompetenz - ein Diskurs in der Krise. In G. Auernheimer (Hrsg.), Interkulturelle Kompetenz und pädagogische Professionalität (S. 35-48). Opladen: Leske + Budrich.

Czollek, L., Perko, G., \& Weinbach, H. (2009). Social Justice, Interkulturelle Öffnung und Diversity als handlungsleitende Prinzipien. In L. Czollek, G. Perko \& H. Weinbach (Hrsg.), Lehrbuch Gender und Queer. Grundlagen, Methoden und Praxisfelder. Weinheim: Juventa.

Dyer, R. (1997). White. London: Routledge.

Eggers, M. M., Kilomba, G., Piesche, P., \& Arndt, S. (Hrsg.). (2005). Mythen, Masken und Subjekte. Kritische Weißseinsforschung in Deutschland. Münster: Unrast.

El-Tayeb, F. (2001). Schwarze Deutsche. Der Diskurs um Rasse und nationale Identität 1890-1933. London, Frankfurt: Campus.

Eppenstein, T. (2003). Einfalt der Vielfalt? Interkulturelle pädagogische Kompetenz in der Migrationsgesellschaft. Frankfurt am Main: Cooperative Verlag.

Foroutan, N. (2013). Hybride Identitäten - Normalisierung, Konfliktfaktor und Ressource in postmigrantischen Gesellschaften. In H. U. Brinkmann \& H.-H. Uslucan (Hrsg.), Dabeisein und Dazugehören. Integration in Deutschland (S. 85-102). Wiesbaden: Springer VS.

Foroutan, N., Karakayali, J., \& Riem, S. (Hrsg.). (2018). Postmigrantische Perspektiven: Ordnungssysteme, Repräsentationen, Kritik. Frankfurt am Main: Campus.

Frankenberg, R. (1993). White women, race matters: the social construction of whiteness. Minneapolis: University of Minnesota Press.

Freise, J. (2005). Interkulturelle Soziale Arbeit. Theoretische Grundlagen - Handlungsansätze - Übungen zum Erwerb interkultureller Kompetenz. Schwalbach im Taunus: Wochenschau.

Fuchs, B., \& Habinger, G. (Hrsg.). (1996). Rassismen \& Feminismen. Differenzen, Machtverhältnisse und Solidarität zwischen Frauen. Wien: Promedia.

Hamburger, F. (2012). Abschied von der Interkulturellen Pädagogik. Plädoyer für einen Wandel sozialpädagogischer Konzepte. Weinheim: Beltz Juventa.

hooks, b (1994b). Teaching to transgress: education as the practice of freedom. New York: Routledge.

Jäger, S. (1993). Kritische Diskursanalyse. Eine Einführung. Duisburg: Diss-Studien.

Jäger, S. (2006). Diskurs und Wissen. Theoretische und methodische Aspekte einer Kritischen Diskursund Dispositivanalyse. In R. Keller, A. Hirseland, W. Schneider \& W. Viehöver (Hrsg.), Theorien und Methoden. Handbuch Sozialwissenschaftliche Diskursanalyse (Bd. 1, 2. Aufl. S. 83-114). Wiesbaden: VS.

Krüger-Potratz, M. (2005). Interkulturelle Bildung. Eine Einführung. Münster: Waxmann.

Langhoff, S. (2011). Die Herkunft spielt keine Rolle - „Postmigrantisches“ Theater im Ballhaus Naunynstraße. https://www.bpb.de/gesellschaft/bildung/kulturelle-bildung/60135/interview-mit-sherminlanghoff. Zugegriffen: 30. März 2020.

Leiprecht, R. (2001). Alltagsrassismus - Eine Untersuchung bei Jugendlichen in Deutschland und den Niederlanden. Münster: Waxmann.

Leiprecht, R. (Hrsg.). (2011). Diversitätsbewusste Soziale Arbeit. Schwalbach im Taunus: Wochenschau Verlag.

Leonardo, Z. (2005). The color of supremacy: beyond the discourse of 'white privilege. In Z. Leonardo (Hrsg.), Critical pedagogy and race (S. 37-52). Malden: Blackwell.

Mamozai, M. (1982). Schwarze Frau, weiße Herrin. Reinbek bei Hamburg: Rowohlt.

Mecheril, P. (2010). Die Ordnung des erziehungswissenschaftlichen Diskurses in der Migrationsgesellschaft. In P. Mecheril, M. Castro Varela, I. Dirim, A. Kalpaka \& C. Melter (Hrsg.), Bachelor/Master Migrationspädagogik (S. 54-76). Weinheim und Basel: Beltz.

Melter, C., \& Mecheril, P. (Hrsg.). (2009). Rassismuskritik. Bd. 1. Schwalbach im Taunus: Wochenschau.

Mignolo, W.D. (2012). Epistemischer Ungehorsam. Rhetorik der Moderne, Logik der Kolonialität und Grammatik der Dekolonialität. Wien: Turia und Kant.

Morrison, T. (1994). Im Dunkeln spielen. Weisse Kultur und literarische Imagination. Essays. Reinbek bei Hamburg: Rowohlt.

Nohl, A.-M. (2006). Konzepte interkultureller Pädagogik. Eine systematische Einführung. Bad Heilbrunn: Klinghardt. 
Oguntoye, K., Opitz, M., \& Schultz, D. (Hrsg.). (1986). Farbe bekennen. Afro-deutsche Frauen auf den Spuren ihrer Geschichte. Frankfurt am Main: Fischer.

Parker, I. (2013). Die diskursanalytische Methode. In U. Flick, E. von Kardorff \& I. Steinke (Hrsg.), Qualitative Forschung. Ein Handbuch (10. Aufl. S. 546-556). Reinbek bei Hamburg: Rowohlt.

Quijano, A. (2016). Kolonialität der Macht, Eurozentrismus und Lateinamerika. Wien, Berlin: Turia+Kant.

Röggla, K. (2012). Critical Whiteness Studies und ihre politischen Handlungsmöglichkeiten für Weiße AntirassistInnen. Wien: mandelbaum kritik \& utopie.

Rommelspacher, B. (1995). Dominanzkultur. Texte zu Fremdheit und Macht. Berlin: Orlanda.

Rosenthal, G. (1995). Erlebte und erzählte Lebensgeschichte. Gestalt und Struktur biographischer Selbstbeschreibungen. Frankfurt am Main: Campus.

Rosenthal, G. (2000). Analyse narrative-biografischer Interviews. In U. Flick, E. von Kardoff \& I. Steinke (Hrsg.), Qualitative Forschung. Ein Handbuch (S. 456-468). Reinbeck bei Hamburg: Rowohlt.

Seshadri-Crooks, K. (2000). Desiring whiteness: a Lacanian analysis of race. London: Routledge.

Staub-Bernasconi, S. (2018). Soziale Arbeit und Menschenrechte. Vom beruflichen Doppelmandat zum professionellen Tripelmandat. Opladen, Berlin und Toronto: Barbara Budrich.

Sullivan, S. (2006). Revealing whiteness. The unconscious habits of racial privilege. Bloomington: Indiana University Press.

Terkessidis, M. (2010). Interkultur. Frankfurt am Main: Suhrkamp.

Tißberger, M. (2005). Uncertainty and method: whiteness, gender and psychoanalysis in Germany. European Journal of Women's Studies. Special Issue: Transformative Methodologies in Feminist Studies, 12(3), 315-328.

Tißberger, M. (2013). Dark Continents und das UnBehagen in der weißen Kultur. Rassismus, Gender und Psychoanalyse aus einer Critical Whiteness-Perspektive. Münster: Unrast.

Tißberger, M. (2016). „Ain’t I a Woman?“ Diversity an der Intersektion von Gender und Rassismus. soziales_kapital, 15, 101-113.

Tißberger, M. (2017). Critical Whiteness. Zur Psychologie hegemonialer Selbstreflexion an der Intersektion von Rassismus und Gender. Wiesbaden: VS.

Tißberger, M. (2017). Critical Whiteness als Praxis im Umgang mit Rassismus, Migration und Intersektionalität in der Sozialen Arbeit. In C. Stark (Hrsg.), Interkulturelle Soziale Arbeit. Forschungsergebnisse 2016 (Bd. 39, S. 8-131). Linz: pro mente.

Tißberger, M. (2019). At the intersection of gender and racism: critical whiteness as a method of hegemonial self-reflection. In C. Bauschke-Urban \& I. Jungwirth (Hrsg.), Gender and diversity studies. European perspectives (S. 149-172). Opladen, Farmington Hills: Barbara Budrich.

Tißberger, M., Dietze, G., Hrzán, D., \& Husmann-Kastein, J. (Hrsg.). (2006). Weiß - Weißsein - Whiteness. Kritische Studien zu Gender und Rassismus. Critical Studies on Gender and Racism. Frankfurt am Main: Peter Lang.

Tißberger, M. et al. (2016). Im Geflecht von Machtverhältnissen. Soziale Arbeit, Rassismus und Intersektionalität aus einer Critical Whiteness-Perspektive. In C. Stark (Hrsg.), Schriften zur Sozialen Arbeit. Forschungsergebnisse 2015 (Bd. 35, S. 105-254). Linz: pro mente.

Wachendorfer, U. (1998). Soziale Konstruktionen von Weiß-Sein. Zum Selbstverständnis Weißer TherapeutInnen und BeraterInnen. In M. Castro Varela, S. Schulze, S. Vogelmann \& A. Weiß (Hrsg.), Suchbewegungen. Interkulturelle Beratung und Therapie (S. 49-60). Tübingen: Deutsche Gesellschaft für Verhaltenstherapie.

Wagner, C. (2017). Öffentliche Institutionen als weiße Räume? Rassismusreproduktion durch ethnisierende Kategorisierungen in einem schweizerischen Sozialamt. Bielefeld: transcript.

Winker, G., \& Degele, N. (2009). Intersektionalität. Zur Analyse sozialer Ungleichheiten. Bielefeld: transcript.

Yancy, G. (2004). Fragments of a social ontology of whiteness. In G. Yancy (Hrsg.), What white looks like. African-American philosophers on the whiteness question (S. 1-23). London: Routledge.

Yildiz, E., \& Hill, M. (Hrsg.). (2015). Nach der Migration. Postmigrantische Perspektiven jenseits der Parallelgesellschaft. Bielefeld: transcript.

Zeller, J. (2000). Kolonialdenkmäler und Geschichtsbewußtsein. Eine Untersuchung der kolonial-deutschen Erinnerungskultur. Frankfurt am Main: IKO-Verlag.

Zimmerer, J., \& Zeller, J. (2003). Völkermord in Deutsch-Südwestafrika. Der Kolonialkrieg (1904-1908) in Namibia und seine Folgen. Berlin: Ch. Links. 